\title{
A Case of Coenurosis in a Wild Rabbit (Lepus sinensis) Caused by Taenia serialis Metacestode in Qinghai Tibetan Plateau Area, China
}

\author{
Xue-Yong Zhang ${ }^{\dagger}$, Ying-Na Jian†', Li-Qing Ma, Xiu-Ping Li, Panagiotis Karanis* \\ State Key Laboratory of Plateau Ecology and Agriculture, Center for Biomedicine and Infectious Diseases, Qinghai University, Qinghai Academy of \\ Animal Sciences and Veterinary Medicine, Qinghai University, Xining 810016, P. R. China
}

\begin{abstract}
Six cystic metacestodes were found in the abdominal muscles of a wild rabbit, Lepus sinensis, in China. The coenurus contained one or more scolices armed with hooklets. Mitochondrial cox 1 (1,623 bp) confirmed 98\% homology with cox 1 of Taenia serialis. This is the first report of $T$. serialis infection in an intermediate host in the Qinghai Tibetan Plateau Area, China.
\end{abstract}

Key words: Taenia serialis, coenurus, rabbit, cox1, China

\section{INTRODUCTION}

Taenia serialis is a rare and neglected taeniid parasite. Adult $T$. serialis is parasitic in the small intestine of the definitive hosts (dogs, foxes, and other canids), and T. serialis larvae develop in the subcutaneous tissues and intramuscular connective tissues of the intermediate hosts (rabbits, hares, rodents, horses, cattle, sheep, goats, and even primates) [1-5]. Coenurosis just is an infection by the metacestode larval stage (coenurus) of $T$. serialis, which usually presents as painless nodules in the parasitic parts of body, so the infection prevalence of $T$. serialis worldwide is likely underestimated $[2,5]$. T. serialis coenurosis is characterized by a large and more tissue-compressing cyst, which contains only a single protoscolex $[1,6]$. Humans cannot be a definitive host for this species of tapeworm, but they can act as an intermediate host. Human T. serialis coenurosis occurs when humans accidentally ingest these eggs, usually in contaminated water and fruits or vegetables [7]. In addition, when T. serialis coenurus is found in the central nervous system (CNS), larval cestode parasites may cause an array of unpleasant symptoms: seizures, ataxia, and further neurological symptoms $[8,9]$. The first proven case of human coenurosis

- Received 16 October 2017, revised 16 February 2018, accepted 20 February 2018. *Corresponding author (panagiotis.karanis@uk-koeln.de)

†Xue-Yong Zhang and Ying-Na Jian contributed equally to this work. (c) 2018, Korean Society for Parasitology and Tropical Medicine This is an Open Access article distributed under the terms of the Creative Commons Attribution Non-Commercial License (http://creativecommons.org/licenses/by-nc/4.0) which permits unrestricted non-commercial use, distribution, and reproduction in any medium, provided the original work is properly cited. due to T. serialis was reported in a 59-year-old French woman by Bonna and colleagues in 1933, and the first infection in the Western Hemisphere was reported in 1950 in a 2-year-old California boy (available in https://web.stanford.edu/group/parasites/ParaSites2005/Coenurosis/). Recently, a T. serialis infection was identified in an African patient (man) from Nigeria with the parasitic larva located subcutaneously on the lower jaw confirmed by molecular tools [1].

Therefore, we performed the present study with morphological and molecular methods to identify the cystic metacestode incidentally recovered from a carcass of a wild rabbit in the Qinghai Tibetan Plateau Area (QTPA), China.

\section{CASE RECORD}

A carcass of 2-year-old male wild rabbit (Lepus sinensis) was found and then dissected by a local veterinarian. A total of 6 cystic metacestodes were recovered in the inner abdominal wall muscles. One of these cysts was transferred in our laboratory and used in this study. The cystic sample was sorted in $70 \%$ ethanol before the molecular study and some part was stored in $10 \%$ buffered formalin for the histopathological examination. The stained slides were observed under a NIKON light microscope, the histological digital photomicrographs were then captured with a computer using the DP 25 digital camera.

The total genomic DNA was extracted from the cysts samples using the QIAamp DNA Mini Kit (Qiagen, Hilden, Ger- 

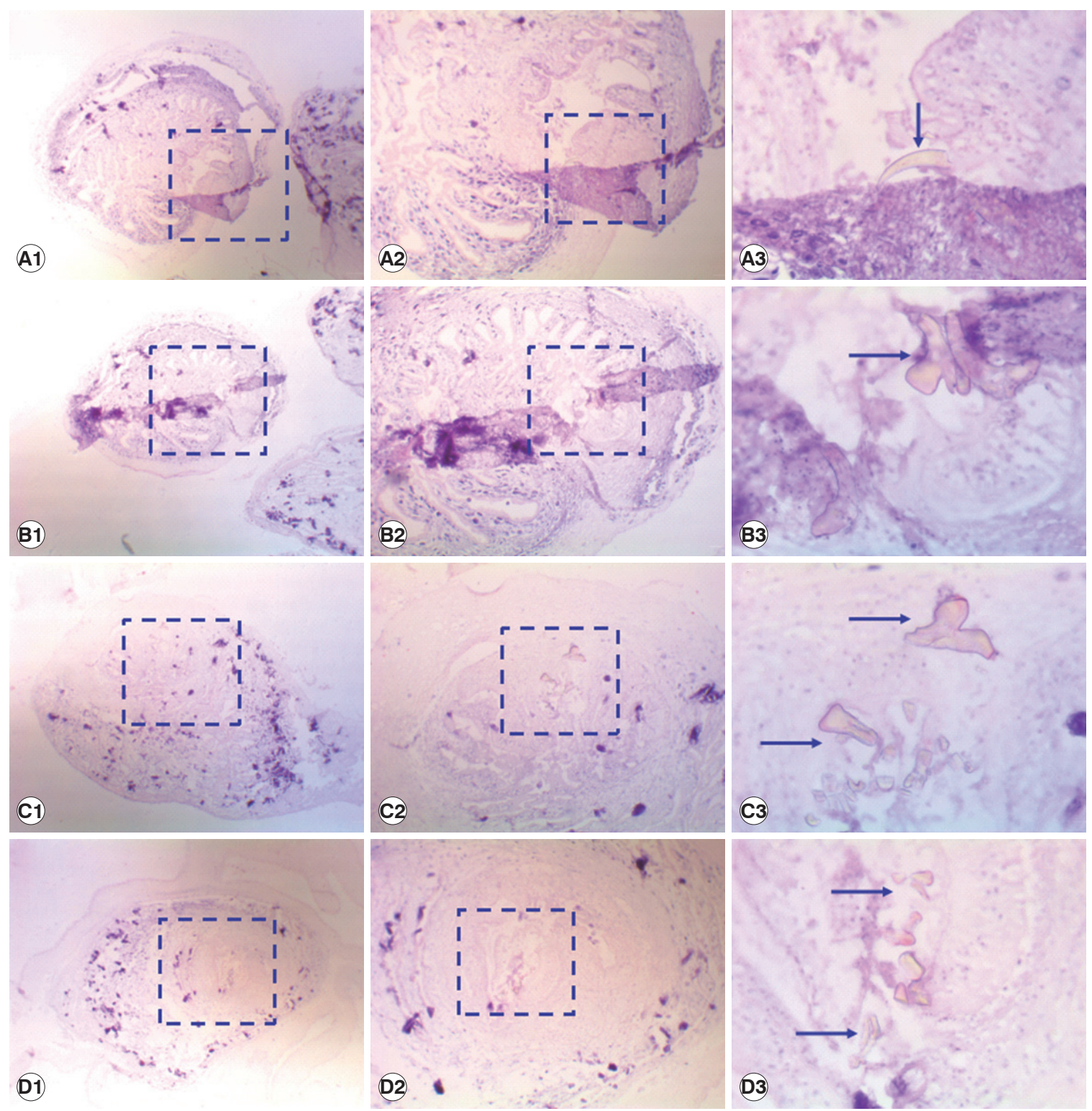

Fig. 1. Coenurus in tissue specimens, stained with hematoxylin and eosin. Images taken at 40×magnification (A1, B1, C1, and D1), $100 \times$ magnification (A2, B2, C2, and D2), $400 \times$ magnification (A3, B3, C3, and D3). Blue arrows point hooklets in the protoscolices.

many), according to the manufacturer's instructions. A PCR was performed to amplify a fragments of the cytochrome $c$ oxidase subunit 1 ( $\operatorname{cox} 1)$ of the mitochondrial genome. The expected lengths obtained after amplification employed the primers T. serialis-cox 1 F: 5' - TTTCGTGTCAATTTGGTTT-3' and T. serialis-cox1R: 5'- GCATGATGCAAAAGGCAAAT-3', the products were $1,844 \mathrm{bp}$. The PCR were performed in standard mixtures of $50 \mu \mathrm{l}$ containing $4 \mu \mathrm{l}$ primer mixtures $(10 \mu \mathrm{M}$ of each primer), $2 \mu \mathrm{dNTP}$ Mix (10 mM of each dNTP), $5 \mu \mathrm{l} \mathrm{10 \times PCR}$ Buffer containing $1.5 \mathrm{mM} \mathrm{MgCl}_{2}, 3 \mu \mathrm{l} 3 \mathrm{mM} \mathrm{MgCl}_{2}, 0.5 \mu \mathrm{l} 5 \mathrm{U}$
HotStar Taq DNA polymerase (Qiagen Gmb H, Hilden, Germany), $2 \mu \mathrm{l}$ DNA and $33.5 \mu \mathrm{l}$ PCR-Grade water. The amplification reactions were run according to the following PCR program: an initial heat-activation step at $95^{\circ} \mathrm{C}$ for $15 \mathrm{~min} ; 35 \mathrm{cy}$ cles of $94^{\circ} \mathrm{C}$ for $45 \mathrm{sec}, 54^{\circ} \mathrm{C}$ for $1 \mathrm{~min}$, and $72^{\circ} \mathrm{C}$ for $2 \mathrm{~min}$; then $72^{\circ} \mathrm{C}$ for $10 \mathrm{~min}$ and a final hold at $4^{\circ} \mathrm{C}$. The amplification PCR products were analyzed using 1.5\% agarose gel containing ethidium bromide $(0.6 \mathrm{mg} / \mathrm{ml})$ and were observed under UV light.

The positive PCR products were directly sequenced by BEI- 


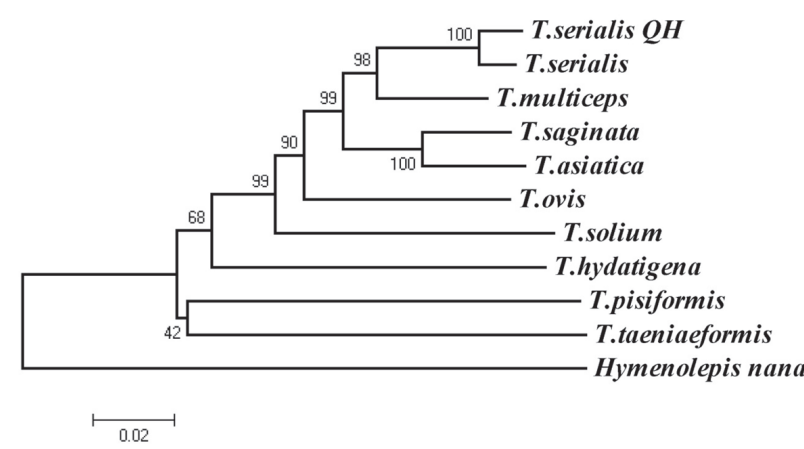

Fig. 2. Phylogenetic tree of 9 species of genus Taenia. T. serialis (AB731674); T. multiceps (JX535570); T. saginata (AB066495); T. asiatica (AF445798); T. ovis (AB731675); T. solium (AB086256); T. hydatigena (FJ518620); T. pisiformis (GU569096); T. taeniaeformis (FJ597547); the sample of this study ("T. serialis QH"). Percentage of replicates is on the node.

JING GENEWIZ Company (Beijing, China). The obtained sequences were then aligned with reference sequences available in the GenBank database using BLAST (http://blast.ncbi.nlm. nih.gov/Blast.cgi). Phylogenetic relationships of Taenia spp. were constructed by the neighbor-joining method, which was performed using the Kimura 2-parameter model and 1,000 bootstrap replicates. Hymenolepis nana (GenBank accession no. KT951722) was used as the outgroup.

The cysts were surrounding a $3 \mathrm{~cm}^{3}$ large cyst, which was composed of a clear, milky and hard membranous bladder and it contained the aggregated scolex of the larval stage of taeniid cestode on the inner aspects. As per Fig. 1 the coenurus cyst was filled with one scolex containing some obvious hooklets surrounded by a fibrous capsule. From the histopathology, the presence of taeniid metacestodes was confirmed and there was a membraneous body with a calcareous thin-wall. The morphological features of the cestode larvae therefore suggested metacestodes of the Taenia species.

The species was identified as T. serialis following the amplification of the fragments of the cox 1 gene, which provided $98 \%$ homology to T. serialis (Genbank accession no. AB731674) when blasted against the NCBI database. The next most closely related species was T. multiceps, with which the Qinghai sample had $94 \%$ nucleotide identity (JX535570) at cox1 gene. The length of cox 1 of Qinghai isolate was 1,623 bp in length and the $\mathrm{G}+\mathrm{C}$ content of which was $30.3 \%$. Phylogenetic reconstruction with published Taeniid cox 1 gene sequences of the mitochondrial genome confirmed that this isolate was more closely related to T. serialis than to any other taeniid species (Fig. 2).

\section{DISCUSSION}

Coenurosis is an uncommon but serious zoonosis, it is often neglected because of its painless nodules in the skin or subcutaneous tissue. However, people have realized the danger of this disease, when it is parasitic in the eyes and the central nervous system. Although it is estimated, that the prevalence of coenurosis in humans is not very high without real "endemic" area. However, T. serialis coenurosis occurs in animals worldwide, with a focus on Europe, Africa, the Middle East and the Americas [5], and the parasite is propagated in a predator-prey cycle that involves definitive hosts (dogs, foxes and other canids) and intermediate hosts (rabbits, hares, and other rodents) [1,2].

In this study, the cysts were found in the muscle of the rabbit, without presentation in the rest of the body or in the eyesexophthalmos [10]. So it was therefore difficult to diagnose coenurosis, but in general the cysts could be identified as Taenia or Multiceps serialis morphologically. In previous studies, the scolex arrangement and the number and size of rostellar hooks were used for the identification [4,11], the hook blades and the morphology of the hook guards were also applied to assist in the identification $[4,12]$. The cysts' morphological identification would be indicative of a T. serialis infection, following the molecular biological confirmation of $T$. serialis from a characteristic cyst in a wild rabbit that could be further strengthened. Previously, some authors declined to assign a determined species in their studies $[13,14]$, while the others attributed coenurosis infection to T. serialis or T. multiceps based on the morphological analysis, which is still not very accurate method to assign the exact parasites species. Morphological identification methods are rapid and convenient assays and it is easy to decipher the results, however, it also has a few shortcomings that are inconsistent. In order to further prove the parasite species, the molecular biology identification was essential. Recently, only one study used molecular method to diagnose a coenurosis in a man from Nigeria and the pathogen identified as T. serialis [1].

In conclusion, this is the first report of T. serialis infection in rabbit in QTPA, China, which indicates the definitive hosts and intermediate hosts of $T$. serialis live in the same environment and area. Further studies should carry out to determine the prevalence of $T$. serialis in definitive hosts and intermediate hosts. Although there is only fragmented record of the occurrence and distribution of $T$. serialis, then which is with a poten- 
tial underestimation of its zoonotic potential and importance to public health concerns.

\section{ACKNOWLEDGMENTS}

This study was supported by the One Thousand Talents Plan of the Chinese Government (no. WQ2013630172) and the National Wool-Purpose Sheep Industry Technology System Project (no. CARS-39-06B).

\section{CONFLICT OF INTEREST}

The authors declare that they have no competing interests.

\section{REFERENCES}

1. Tappe D, Berkholz J, Mahlke U, Lobeck H, Nagel T, Haeupler A, Muntau B, Racz P, Poppert S. Molecular identification of zoonotic tissue-invasive tapeworm larvae other than Taenia solium in suspected human cysticercosis cases. J Clin Microbiol 2016; 54 : 172-174.

2. Ing MB, Schantz PM, Turner JA. Human coenurosis in North America: case reports and review. Clin Infect Dis 1998; 27: 519523.

3. Hough I. Subcutaneous larval Taenia serialis in a ring-tailed possum (Pseudocheirus peregrinus). Aust Vet J 2000; 78: 468.

4. Schneider-Crease IA, Snyder-Mackler N, Jarvey JC, Bergman TJ. Molecular identification of Taenia serialis coenurosis in a wild Ethiopian gelada (Theropithecus gelada). Vet Parasitol 2013; 198 : 240-243.
5. Schneider-Crease I, Griffin RH, Gomery MA, Dorny P, Noh JC, Handali S, Chastain HM, Wilkins PP, Nunn CL, Snyder-Mackler $\mathrm{N}$, Beehner JC, Bergman TJ. Identifying wildlife reservoirs of neglected taeniid tapeworms: Non-invasive diagnosis of endemic Taenia serialis infection in a wild primate population. PLoS Negl Trop Dis 2017; 11: e0005709.

6. Jull P, Browne E, Boufana BS, Schoniger S, Davies E. Cerebral coenurosis in a cat caused by Taenia serialis: neurological, magnetic resonance imaging and pathological features. J Feline Med Surg 2012; 14: 646-649.

7. Benger A, Rennie RP, Roberts JT, Thornley JH, Scholten T. A human coenurus infection in Canada. Am J Trop Med Hyg 1981; 30: 638-644.

8. Coyle CM, Mahanty S, Zunt JR, Wallin MT, Cantey PT, White AC Jr, O'Neal SE, Serpa JA, Southern PM, Wilkins P, McCarthy AE, Higgs ES, Nash TE. Neurocysticercosis: neglected but not forgotten. PLoS Negl Trop Dis 2012; 6: e1500.

9. Garcia HH, Nash TE, Del Brutto OH. Clinical symptoms, diagnosis, and treatment of neurocysticercosis. Lancet Neurol 2014; 13: 1202-1215.

10. O'Reilly A, McCowan C, Hardman C, Stanley R. Taenia serialis causing exophthalmos in a pet rabbit. Vet Ophthalmol 2002; 5: 227-230.

11. Clark JD. Coenurosis in a gelada baboon (Theropithecus gelada). J Am Vet Med Assoc 1969; 155: 1258-1263.

12. Meyer MC. Coenuriasis in varying hare in Maine, with remarks on the validity of Multiceps serialis. Trans Am Microsc Soc 1955; 74: 163-169.

13. Malomo A, Ogunniyi J, Ogunniyi A, Akang EE, Shokunbi MT. Coenurosis of the central nervous system in a Nigerian. Trop Geogr Med 1990; 42: 280-282.

14. Templeton AC. Human coenurus infection. A report of 14 cases from Uganda. Trans R Soc Trop Med Hyg 1968; 62: 251-255. 